\title{
GRUPOS DE AYUDA MUTUA: UNA RESPUESTA ALTERNATIVA EN LA PRÁCTICA DEL TRABAJO SOCIAL
}

\section{SONIA RÓDENAS PICARDAT}

Trabajadora Social. Coordinadora de la Sede de Elche de la Asociación Ciudadana contra el SIDA de la provincia de Alicante (A.Co.S.P.A.).

\section{INTRODUCCIÓN}

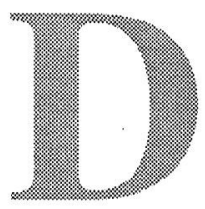

esde hace varios años vengo aplicando en diferentes áreas del trabajo social, como son las drogodependencias y el trabajo con enfermos afectados por el VIH/SIDA, un tipo de grupo que he podido constatar en mis encuentros con compañeras/os de la profesión, resulta por todos conocido, pero poco empleado por no saber exactamente en qué consiste. Así, y acogiéndome al nombre de la revista que publica la Escuela de Trabajo Social de la Universidad de Alicante, he decidido escribir este artículo en base a mi experiencia y a la teoría a partir de la cual me he ido formando, para así poder brindar a los profesionales que lo deseen una respuesta ALTERNATIVA a los problemas y necesidades que presentan algunos de nuestros usuarios.

La evolución de nuestra profesión ha hecho que, todavía hoy en día, el enfoque principalmente individual tenga un peso considerable en la práctica del trabajo social. Y aunque progresivamente, e influenciados por las recientes circunstancias que nos rodean y por las nuevas corrientes provenientes de otros países, de otras profesiones y de los propios trabajadores sociales, el segundo nivel de intervención, es decir, el grupal, se emplea cada vez más, aún no se recurre al mismo lo que se debería y se podría. Una muestra de ello es la formación en los campos de prácticas donde los alumnos suelen intervenir en su gran mayoría en el primer nivel. Con todo ello, el resultado es que los trabajadores sociales disponen de una «herramienta» que en numerosos casos resulta muy eficaz, aunque poco empleada. Y dentro de este tipo de respuesta se encuentra el GAM.

Y el otro motivo, ya dentro de esta clase de grupo, por el cual los profesionales suelen tener dificultades a la hora de llevarlo a la prácti$\mathrm{ca}$, es que tratan de hacerlo de un modo terapéutico cuando su finalidad no es ésta, o de incluirse ellos en el mismo cuando su lugar no es ése. Sin embargo, me gustaría aclarar que no por no participar directa- 
mente en los GAM, éstos dejan de tener una relación muy estrecha con nuestra profesión como quedará aclarado tras la lectura del artículo.

\section{2. ¿QUÉ ES UN GRUPO DE AYUDA MUTUA?}

Como cualquier grupo, el GAM es una pequeña reunión de personas. Pero en este caso, sus miembros se reúnen de forma voluntaria y libre, movidos por la necesidad de dar respuesta o encontrar una solución a un problema compartido por todos ellos, de afrontar y superar una misma situación conflictiva, o de lograr cambios personales y/o sociales. En este tipo de grupo, no está presente ningún profesional externo (al menos que el grupo lo llame de forma puntual para pedirle algún tipo de información técnica). Sus componentes se ayudan los unos a los otros a través de las interacciones que, entre ellos y en el marco del grupo, se producen. De ello deducimos que este tipo de grupo facilita el crecimiento individual de sus miembros en cuanto a asunción de responsabilidades y adquisición de nuevos valores, ya que facilita la toma de decisiones pero nunca decide por el otro o le da la respuesta a su problema. Por otro lado, sus miembros aprenden a escuchar, a valorar los problemas de los demás y a ayudarles. Los GAM proporcionan principalmente apoyo emocional y, ocasionalmente, ma- . terial. La autoayuda es pues un estilo de entender la comunicación.

\section{3. ¿CÓMO FUNCIONA?}

Los GAM están formados por un grupo de personas que se reúnen sin la presencia de un profesional. Y ello porque una de las premisas de los GAM es que todos sus miembros se sitúen al mismo nivel y no sean dirigidos. La presencia de un profesional, aunque éste quisiera situarse a la misma altura que los demás miembros, impediría el correcto funcionamiento del grupo, puesto que generalmente los otros componentes piensan que sus aportaciones pesan más y tienen tendencia a dirigirse a él y no al conjunto de los asistentes. Ello sin olvidar que, en la práctica, al profesional le es difícil desligarse de su papel directivo.

Los grupos de ayuda mutua también pueden encontrarse bajo el nombre de:

- Grupo de autoayuda.

- Grupo de autoapoyo.

- Grupo de soporte. 
Sin embargo, para poder funcionar correctamente, hace falta la presencia de una persona que posibilite la marcha del grupo. De entre los miembros del grupo, y elegido por éste de forma democrática, surge la figura del facilitador cuyas funciones son:

a) La de moderador, limando las asperezas que puedan existir entre las personas y potenciando la participación de todos los miembros; a veces, ciertas personas más tímidas o reservadas no participan y ello no es conveniente ni para ellas, que no pueden compartir su problema, desahogarse y tratar de encontrar una solución al mismo, objetivos últimos de los GAM, ni para los demás, porque no sólo no reciben ayuda por parte de esas personas sino que pueden llegar a experimentar una sensación de vacío, de «desnudez», de entrega sin recibir nada a cambio. Es decir, en esta clase de grupos, la base, como ya hemos visto, es la interacción entre sus miembros. Si, por algún motivo, la retroalimentación resulta deficitaria, ello perjudica gravemente el buen funcionamiento del grupo.

Esta cuestión es delicada y requiere de una gran habilidad por parte del que lleva el grupo, pues tampoco se puede forzar a las personas a hablar. Una buena forma de «enganchar» a los miembros no participativos es la de hacerlo a través de la comunicación no verbal. También hay que comprender que cada personas tiene su momento y que hay días mejores que otros. Pero siempre hay que tener en cuenta que el GAM se ha de llevar a cabo entre todos.

Puede entonces surgir la duda de cómo una persona, que no ha recibido una formación profesional, puede dirigir convenientemente el grupo. Por una parte, y al ser elegido por el propio grupo, se supone que la figura del facilitador surge de entre uno de sus líderes, lo cual facilita la tarea. Por otra, lo idóneo es que esa persona haya recibido una formación previa por parte de la organización a la que pertenece y/o a través de cursos de formación de voluntariado.

b) La de nexo de unión entre el interior y el exterior del grupo, invitando a los profesionales y/o haciendo posible que entre la información que el grupo necesite y demande o que salga aquella que facilite las necesidades del grupo, posibilitando el contacto de este grupo con otros, haciendo que el grupo se dé a conocer para que otros interesados sepan de su existencia o facilitando la llegada de nuevos componentes.

Con relación a este último aspecto, diremos que, puesto que la confianza es la base de estos grupos, hay que ayudar a ese nuevo miembro a romper el hielo y a integrarse, pero también hay que lograr que el propio grupo se abra a él y le deje un espacio. Y ello no siempre es fácil. Por un lado, porque las personas ya están acomodadas al grupo conformado hasta el momento y readaptarse cuesta y, por otro lado, porque supone un breve retroceso y una repetición cada vez que uno nuevo se incorpora. 
Si bien, y como ya mencioné con anterioridad, el grupo puede llegar a prestar una ayuda material a sus miembros, su principal función es el apoyo emocional. Este se puede dar de varias formas:

a) A través del desahogo y de la liberación de sentimientos. En la mayoría de los problemas sociales pesa sobre los afectados una fuerte discriminación, además de la barreras físicas y psicológicas, que causa el aislamiento de muchos de ellos, haciendo que vivan su situación en silencio y soledad. Incluso el hallar a personas que le escuchen a uno no sirve realmente de apoyo cuando éstas son ajenas a la problemática. EI GAM proporciona pues un lugar donde compartir los miedos, las angustias, las depresiones, las rabias, los odios, pero también las alegrías, la esperanza...

b) A través de la técnica del espejo (Orsi, J.F. y Pinto Martín, E., 1991). Cuando una persona tiene un problema, le cuesta aclarar sus ideas con respecto al mismo, encontrar posibles soluciones a éste y remontar los estados emocionales por los que pasa. Sin embargo, cuando el que atraviesa el conflicto es otro, resulta más fácil ver con claridad en qué consiste éste y, por lo tanto, hallarle una solución. En el caso de los GAM, lo que cuenta la otra persona es lo que vive uno mismo y así, al encontrar una respuesta al problema ajeno, generalmente lo estamos haciendo al nuestro propio.

Pero la ayuda no debe quedarse aquí. Puesto que la base del funcionamiento de los GAM son las interacciones del grupo, ante la narración del otro se produce una retroalimentación que consiste en la devolución de la ayuda a éste a través de frases, preguntas y cuestionamientos que le faciliten la aclaración de sus ideas. Pero nunca se debe dar consejos o expresar lo que uno haría en el lugar del otro, puesto que no lo está. Para ello existen una serie de técnicas que expondré más adelante. El parafrasear, por ejemplo, ayuda al otro a ver sus paradojas e incongruencias, o simplemente le muestra que los otros componentes del grupo le escuchan y prestan atención.

La técnica del espejo facilita la racionalización de sentimientos como el egocentrismo, la negación, la rabia, el odio, las ideas irracionales...

c) El funcionamiento del grupo no se limita a los espacios físico, temporal y problemático. La ayuda mutua va más allá, prestándose también entre sus miembros durante el intervalo existente entre reunión y reunión. Si uno de los miembros se encuentra mal un día en que no hay GAM, tiene a quien recurrir para hablar de lo que le sucede.

En contrapartida, a lo largo de las sesiones del GAM, no siempre se tiene por qué hablar del problema que los reúne. El grupo es también un lugar de encuentro social donde rehacer las relaciones que muchos de sus miembros habían perdido. También se organizan actividades de ocio y tiempo libre. 
Para finalizar este apartado, voy a enumerar algunas de las técnicas y condiciones que facilitan la evolución de los grupos (Proyecto Hombre 1994):

- No olvidar que el otro es el protagonista de su propia historia. Debemos pues evitar dar consejos y soluciones.

- Tener en cuenta que uno solo no puede.

- La honestidad y la claridad son importantes.

- Es importante hablar desde el presente (el pasado no se puede cambiar, pero sí se puede actuar en el presente para que cambie el futuro).

- Empatizar, identificarse con el otro.

- El silencio interior (para escuchar al otro sin prejuicios ni ideas preconcebidas).

- Diferenciar entre sentirse bien /mal y estar bien/mal.

- La autoayuda va dirigida al aprendizaje desde el conocerse a uno mismo: qué se quiere cambiar y cómo.

- Nunca se debe preguntar «por qué».

- Nunca se debe interpretar.

- Centrarse siempre en los sentimientos.

- Nunca hay que preguntar por la responsabilidad del problema.

- La base de los GAM es la escucha y existen ejercicios sencillos para practicarla con el grupo, como, por ejemplo, «radio macuto» (Vargas, Bustillos y Marfán, 1993).

\section{TIPOS DE GAM}

Podemos situar los orígenes de los GAM en el entorno psiquiátrico y psicosocial y, más exactamente, en los grupos formados por personas con problemas de alcoholismo, toxicomanías o mentales graves (Orsi, J.F. y Pinto Marti, E., 1991). Pero en los últimos años esta tipología se ha ampliado considerablemente. He aquí dos clasificaciones diferentes de los GAM (Roca y Villalbi, 1993):

\subsection{Según sus componentes}

- Los formados por las personas que viven directamente el problema: los toxicómanos, los infectados y/o enfermos de VIH/SIDA...

- Los compuestos por aquellos que padecen indirectamente el problema: los familiares y personas más allegadas.

- Los integrados por los profesionales: éste es uno de los tipos más recientes, pero cada vez más practicado, ya que ciertos colectivos enfrentados a lo largo de toda su vida profesional a situaciones 
estresantes pueden necesitarlos para poder acometer eficazmente su labor; pongamos, por ejemplo, las enfermeras de enfermos terminales, los cuidadores, los trabajadores sociales, los psicólogos...

$\mathrm{Si}$ ello es factible, lo ideal es formar los tres GAM por separado, pero, si no hay suficientes personas para ello, se organiza uno sólo a donde acudan tanto las personas que viven el problema sobre sí mismas, como sus familiares, amigos... En este caso, el facilitador y los miembros del grupo tienen que tener en cuenta que los problemas vividos por ambos colectivos no siempre son los mismos y que, además, la presencia de un conocido puede inhibir o dificultar la participación y la liberación del otro miembro.

\subsection{Según la problemática}

- Algunos grupos se centran alrededor de una enfermedad crónica: es el caso de la Esclereosis Lateral Amiotrófica (ELA), el Síndrome de Inmunodeficiencia Adquirida (SIDA)...

- Otros agrupan a personas con problemas de comportamiento personal: alcohol, droga, obesidad por comer de forma compulsiva, anorexia, juego..., a los cuales les resulta muy difícil realizar el cambio aisladamente.

- Ultimamente han proliferado los grupos de personas con problemas psicosociales: separación de la pareja, pérdida de un hijo, problemas creados por la vejez, personas recién salidas del hospital o de la cárcel...

- O debido a problemas generados por la propia sociedad: marginación debida a la homosexualidad, minorías étnicas, inmigración...

\section{OBJETIVOS DEL GAM}

Cuando un GAM se reúne por primera vez es conveniente que cada miembro se presente y exprese sus expectativas. A continuación, el grupo va fijando unas reglas mínimas para su funcionamiento que se van ampliando, confirmando o refutando con el tiempo y establece sus objetivos. De estos objetivos dependerán sus actividades. A grandes rasgos, y en función de lo expuesto hasta el momento, podemos perfilar cuatro objetivos que suelen ser comunes a todos los GAM (AA.VV., 1994):

a) Facilitar un apoyo psicológico a los miembros del grupo (autovalorarse, desahogarse, liberarse, apoyarse mutuamente, ser aceptado, ser comprendido...). 
b) Rehacer y potenciar las relaciones sociales de los afectados (salir del aislamiento, conocer a otras personas, realizar actividades de ocio y tiempo libre...).

c) Informar sobre los recursos existentes, sobre la problemática en sí, sobre posibles actividades...

d) Servir (si fuera necesario) de grupo de presión (reivindicación de los derechos, denuncia de las discriminaciones...).

En sesiones sucesivas es conveniente empezar el grupo haciendo una ronda breve donde cada uno diga cómo está, lo que querría hacer en el grupo. Esto pretende ser una introducción para abrir el grupo.

\section{ACTIVIDADES}

Como hemos visto, las actividades están en función de los objetivos. Estas también las fija el grupo. A título de ejemplo citaremos las siguientes:

1. En relación al objetivo psicológico:

- Hablar en el grupo.

- Charlar fuera de las sesiones.

- Visitas hospitalarias y a domicilio.

- Visitas a la cárcel.

- Ocio y tiempo libre.

- En cuanto al objetivo de las relaciones sociales:

Principalmente, actividades de ocio y tiempo libre y charlar fuera de las sesiones.

2. En relación al objetivo de recursos y actividades:

- Sesiones de video.

- Invitación de expertos sobre el tema que demande el grupo.

- Aportar documentación.

3. Y, por último, el objetivo relativo al grupo de presión:

- Cartas.

- Medios de comunicación.

- Mesas divulgativas.

- Conferencias.

- Manifestaciones...

\section{TÉCNICAS}

La base del soporte es que la mayoría de las personas son capaces de resolver sus problemas si se les da la oportunidad. El papel del facilitador no es proporcionar las soluciones sino ayudar a las perso- 
nas a que encuentren sus propias respuestas (aunque esto no es exclusivo del facilitador). Y esto se consigue utilizando unas ciertas técnicas que ayudan a clarificar los pensamientos y los sentimientos y a explorar las diferentes opciones y soluciones, y que son:

- La técnica del espejo.

- Parafrasear: confrontar, empatizar, clarificar, valorar la comprensión.

- Empleo de preguntas abiertas: preguntas reflexivas.

- Ventilación: trabajar los sentimientos.

- Focalizar las emociones ligadas al conflicto para poder afrontar el problema (que sean sentimientos y no pensamientos: "me siento", "estoy"...).

- Parafrasear el sentimiento expresado.

- Reflejar los sentimientos (sobre todo, los no verbales, pero sin interpretar).

- Definir y clarificar los sentimientos (¿qué significan para las personas?).

- Reconocer los sentimientos.

- Exteriorizar los sentimientos.

- Escucha activa.

- Observación.

- Técnicas participativas (Vargas, Bustillos, Marfán, 1993) y para la toma de decisiones, la resolución de conflictos, la toma de confianza... (AA.VV., 1994).

- Técnicas con soporte auditivo o visual (videos, ilustraciones, escucha...).

- Dinámicas de animación.

- Técnicas de evaluación (a través del informe del grupo).

\section{ROLES}

En este apartado, me voy a referir a los roles explícitos y fijados por el grupo y no a los implícitos que surgen de cualquier relación humana, puesto que el artículo no trata sobre la teoría de los grupos en general sino más bien sobre la práctica de los GAM en concreto.

En éstos, todos los miembros han de situarse al mismo nivel. Debemos destacar, sin embargo, el rol del facilitador que ya describimos con anterioridad. En cuanto a los otros roles, por tratarse de unos grupos donde deben compartirse las responsabilidades, es conveniente que aquellos que conlleven un mayor peso sean rotatorios. De lo contrario, las personas que los ocupan suelen cansarse y abandonar el grupo. Por otro lado, hay que destacar que es conveniente que cada miembro ten- 
ga un rol (el que busca financiación para el grupo, el que organiza las actividades, etc.).

También resulta importante fijar el rol de escucha activa (sus funciones son: no interrumpir, mostrarse interesado y atento, nunca juzgar, no ofrecer soluciones ni consejos, respetar el silencio, hacer preguntas abiertas...).

\section{NORMAS}

También éstas son establecidas por el grupo:

- El acceso al grupo es totalmente voluntario y libre. De hecho, los principios que suelen inspirar a este tipo de grupo son los de la cooperación y la ayuda mutua.

- Además, el grupo ha de ser abierto, es decir, que no puede negar de antemano la asistencia al mismo a alguien en concreto. Ello tiene como consecuencia más directa que los GAM suelen ser rotatorios. El facilitador y los miembros del grupo deberán tenerlo en cuenta a la hora de establecer un clima de confianza cuando los miembros del grupo son muy cambiantes, o, a lo contrario, cuando se trata de personas asiduas, ayudar a la incorporación de un nuevo miembro.

- Se trata de grupos con un funcionamiento democrático.

- Debe crearse una atmósfera de confianza.

- Deben fijarse las tareas de todos entre todos.

- Normas básicas:

- No violencia.

- No consumo de drogas (incluidos tabaco y alcohol).

- Puntualidad.

- Confidencialidad (secreto de grupo).

- Respetar a todos los miembros (eso implica dejar que todos participen y escuchar lo que dicen).

- No juzgar.

\section{OTROS DATOS SOBRE LA ESTRUCTURA DE LOS GAM}

En cuanto al número de participantes, con tres personas ya se puede iniciar un GAM. Como en los demás tipos de grupos, el número ideal oscila alrededor de los 10/12 miembros. Aunque tal vez al principio sea mejor un número más reducido para permitir una mayor participación de sus miembros y, así, una mayor intimidad y compenetración y poco a poco ir creciendo. El tope se encuentra a partir del momento en que no todos los miembros del grupo tengan participación 
durante las sesiones. En este caso, se puede subdividir según diferentes criterios, y siempre intentando cubrir las principales necesidades de las personas. En algunos casos, el horario o turno de trabajo puede ser un criterio, pero también pueden serlo los sociológicos como la edad, la relación con el problema...

El día y la hora están en función del grupo. Aunque sí podemos especificar que la duración suele oscilar entre una hora y dos. La periodicidad más frecuente es la de una vez por semana, si bien algunos GAM se reúnen dos veces por semana y otros cada quince días. Espaciarlos más dificulta la consecución de ciertos objetivos del GAM.

Es conveniente que el lugar reúna ciertos requisitos:

- Estabilidad.

- Confortabilidad.

- Comodidad para todos.

- Neutralidad.

- Facilidad de intimidad.

- No vinculado.

El GAM, contrariamente a la mayoría de los otros grupos, tiene un principio para su miembro pero no tiene un fin claro y preestablecido. La persona puede pasarse años en él si se encuentra a gusto.

\section{EL PAPEL DEL TRABAJADOR SOCIAL EN RELACIÓN A LOS GAM}

Tras la exposición hasta ahora desarrollada, ha quedado clara la ausencia del trabajador social en los GAM, si bien su función queda estrechamente relacionada con los mismos:

- Normalmente, los trabajadores sociales suelen estar al inicio de los GAM (unas cuatro sesiones) informando de que su estancia es ocasional, con el fin de orientar al grupo y al facilitador en sus comienzos. En cuanto pueda, el profesional debe abandonar el grupo.

- Como técnico al que se consulta de forma puntual sobre algo relacionado con la profesión (técnicas de duelo, de relación de ayuda, de dinamización, problemas sociales...).

- Cuando surgen problemas en el seno del grupo que los miembros por sí solos no pueden solucionar, puede ser llamado como moderador.

- Ha de conocer muy bien cuáles son los problemas y las necesidades a las que dan respuesta los GAM para poder derivar a los usuarios que lo necesiten. Por lo general, en las asociaciones e instituciones que cuentan con GAM, un gran número de personas dejan de ser derivadas a terapias individuales o a grupos terapéuticos, pues, generalmente, con los GAM es suficiente para resolver la situación en la que se en- 
cuentran (depresión, soledad...).

- Es uno de los profesionales que forma a los facilitadores en los cursos de formación del voluntariado.

- Cuando su puesto lo requiere, el GAM resulta de una gran utilidad para los trabajadores sociales como participantes en el mismo.

\section{BIBLIOGRAFÍA}

AA.VV. "La alternativa del juego II - Juegos y dinámicas de educación para la paz". Seminario de Educación para la Paz. Asociación Pro Derechos Humanos. Editorial Catarata. Madrid, 1994; 11, 15-42, 95-116 y 181-222. AA.VV. "La conducta humana en el medio social" Enfoque sistémico de la sociedad. Edita Gedisa. Barcelona, 1994; 207.

BEAL, G.M. y otros: Conducción y acción dinámica del grupo. Kapelusz. Buenos Aires, 1964.

HAIMAN, F.: Dinámica de grupos. Teoría y práctica. Limusa. Barcelona, 1976.

KONOPKA, G.: Trabajo Social de Grupo. Euroamérica. Madrid, 1968.

MUCCHIELLI, R.: La dinámica de los grupos. Ibérico Europa de Ediciones. Madrid, 1977.

ORSI, J.F. y PINTO MARTÍN, E. (traductores): "Liberar la prevención". Edita Asociación Proyecto Hombre. Madrid, enero 1991; 22-184 y 209-282.

PAGES, M.: La vida afectiva de los grupos. Hogar del libro. Barcelona, 1988.

ROCA, F. y VILLALBI, J.R.: Manual para el funcionamiento de grupos de ayuda mutua. Fondo de Publicaciones del Gobierno de Navarra. Pamplona, 1993; 11.

TSCHORNE, P.: La dinámica de grupo aplicada al Trabajo Social. Obelisco. Barcelona, 1990.

VARGAS, L.; BUSTILLOS, G. y MARFÁN, M.: Técnicas participativas para la educación popular. Editorial Popular. Madrid, 1993; 209-228. 